\title{
Preface to the Special Topic on Water Science
}

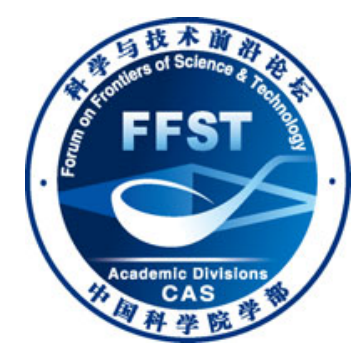

The enormous abundance of water on Earth and its pivotal significance to our life are well known, but the many anomalies of such a matter of very simple composition, and the difficulties for scientists to access them, however, are not fully recognized. In fact, rarely any result over the understanding of a property of water is free of debate.

Water science is a traditional multidisciplinary research topic. In recent years, various working groups from the institutes of Chinese Academy of Sciences and some universities of China have devoted their efforts toward this intriguing and challenging enterprise, and now they all begin to gain some authority in the international circle of water science. In this special topic we publish five review articles, all of which are based on the results of the corresponding research groups. The article contributed by Prof. FANG HaiPing and his coworkers reviews some of recent advances in the atom-level pictures of interfacial water which exhibits an ordered character on various solid surfaces at room temperature or cryogenic temperature. Special focus is devoted to the wetting phenomenon of "ordered water monolayer that does not completely wet water" and the underlying mechanism. Prof. XU LiMei et al. present the experimental and theoretical progresses on the study of the liquid-liquid phase transition in water, including discussion on the first-order liquid-liquid phase transition in supercooled water and the detection of liquid-liquid critical point. In the article authored by Prof. WU XingLong and his coworkers, the quantum confinement effect and the stability of 3C-SiC nanocrystals in aqueous solution are discussed, aiming at a more efficient splitting of water. The physical properties and the transport of confined bulk water are the long-term concern for the work group of Prof. WEN WeiJia, and the investigation over this topic is scrutinized with regard to understanding water transport in plant and groundwater extraction. A brief review by Prof. JIANG Lei et al. covers the recent progress in wettability manipulation in nanoconfined environment, water transportation through biological and artificial nanochannels, and the wettability control of smart nanofluidic gating systems.

It is our great pleasure to have served as the guest editors of this special topic, and we are very grateful to all the authors who have contributed to both the accomplishment of relevant researches and the compiling of the review articles. We expect more marvelous works on water science to appear in China, and we are also looking forward to the editing of a voluminous special issue over water science in the near future.

CAO ZeXian \& YANG GuoZhen Guest Editors, Institute of Physics, Chinese Academy of Sciences 